\title{
Management of Toxicity Induced by Anti-EGFR Therapy in Metastatic Colorectal Cancer
}

\author{
Christian Rolfo • Giuseppe Bronte • Francesco Passiglia • \\ Konstantinos Papadimitriou • Antonio Russo • Marc Peeters
}

Published online: 8 June 2013

(C) Springer Science+Business Media New York 2013

\begin{abstract}
Use of anti-epidermal growth factor receptor (anti-EGFR) agents has yielded significant advances in the treatment of patients with metastatic colorectal cancer. In fact these drugs, which include the monoclonal antibodies cetuximab and panitumumab, can be delivered both as a single agent and in combination with chemotherapy, achieving better survival and quality of life and in some cases also resectability of metastases. However, these agents can result in the development of toxicities that are usually different from those observed with chemotherapy alone. For the management of these adverse effects, proper knowledge is mandatory. Skin toxicity is the most frequent adverse effect. Other toxicities can be observed, such as hypomagnesemia, gastrointestinal toxicity, and thromboembolic events. Severe infusion reactions can be life-threatening. For these reasons a review of anti-EGFR-drug-related toxicity is useful for clinical practice.
\end{abstract}

\footnotetext{
C. Rolfo $\cdot$ K. Papadimitriou $\cdot$ M. Peeters $(\triangle)$

Oncology Department, Antwerp University Hospital

and Multidisciplinary Oncology Center Antwerp (MOCA),

Wilrijkstraat 10, 2650 Edegem, Belgium

e-mail: marc.peeters@uza.be

C. Rolfo

e-mail: christian.rolfo@uza.be

K. Papadimitriou

e-mail: konstantinos.papadimitriou@uza.be

G. Bronte $\cdot$ F. Passiglia $\cdot$ A. Russo

Medical Oncology, Department of Surgical and Oncology

Sciences, University of Palermo, Palermo, Italy

G. Bronte

e-mail: giuseppe.bronte@unipa.it

F. Passiglia

e-mail: francesco.passiglia@unipa.it

A. Russo

e-mail: antonio.russo@usa.net
}

Keywords Toxicity · Epidermal growth factor receptor . Anti-epidermal growth factor receptor · Cetuximab . Panitumumab · Antibody $\cdot$ Metastatic $\cdot$ Colon $\cdot$ Rectum Cancer · Treatment · Skin · Rash · Cutaneous · Pruritus . Xerosis $\cdot$ Paronychia $\cdot$ Hypomagnesemia $\cdot$ Magnesium . Gastrointestinal · Diarrhea · Infusion · Reaction · Pulmonary · Fibrosis

\section{Introduction}

Colorectal cancer (CRC) is one of the leading causes of cancerrelated deaths. In recent decades the integration of different therapeutic strategies, such as surgery, radiation therapy, and systemic therapy, has yielded improved overall survival and quality of life. Systemic therapy includes both chemotherapeutic regimens and targeted drugs. However, the introduction of new targeted drugs has resulted in the development of specific toxicities and a radically different tolerability profile compared with chemotherapy. Because cytotoxic agents are used for cancer therapy, all early-phase studies were designed to identify the maximum tolerated dose and the dose-limiting toxicities. These parameters are based on the close relationship between the dose and the toxicity frequency and magnitude. In contrast, the side effects caused by targeted drugs seem to be influenced by the dose, but a clear relationship has not been defined.

The anti-epidermal growth factor receptor (anti-EGFR) agents used in CRC treatment are the monoclonal antibodies cetuximab and panitumumab. For these drugs, the commonest side effects are related to the expression of EGFR in normal tissues. Skin, the gastrointestinal tract, and the kidney are all tissues where EGFR is normally expressed.

Since skin rash, diarrhea, and hypomagnesemia, which arise during anti-EGFR treatment, seem to be different from those induced by chemotherapy, this review gathers the most recent knowledge regarding this topic. Other side effects such as pulmonary and thromboembolic events are rare with these 
agents, even though they are more associated with other EGFR inhibitors such as tyrosine kinase inhibitors, which are used in treatment of other malignancies. For each toxicity, we report a clinical description, the known pharmacological mechanisms, and the management. A summary of the biological mechanism of the anti-EGFR inhibitors is also included.

\section{Skin Toxicity}

Skin toxicity is the commonest side effect observed during the treatment with anti-EGFR agents. It includes several signs and symptoms, such as papulopustular rash, xerosis/fissures, pruritus, paronychia, hair changes, and mucositis. The incidence ranges from $30 \%$ for mucositis to $80 \%$ for skin rash (Fig. 1). Although less than $20 \%$ of patients have severe symptoms [1], skin toxicity is visible, often causes physical and emotional discomfort, resulting in a significant impact on quality of life [2••], and may lead to dose reduction in $60 \%$ of patients or discontinuation of treatment in $32 \%$ of them [3], with subsequent worse clinical outcomes and an increase of management costs.

\section{Pharmacological Mechanisms}

The exact mechanism of skin toxicity related to EGFR inhibitors is not clearly understood. The coincident inhibition of receptor activity in tissue that depends on EGFR signaling for normal functions seems to play a critical role in rash development, resulting in impairment of keratinocyte growth and differentiation and increased apoptosis [4]. There is some evidence in the literature that these agents may alter the immune system. More recently, a preclinical model showed the role of tumor necrosis factor $\alpha$ (TNF- $\alpha$ ) and interleukin-1 in the development of skin rash associated with EGFR inhibitors, and it suggested a possible therapeutic role for antiTNF- $\alpha$ agents [5]. Several studies have aimed to identify histological and immunohistochemical features of the skin during therapy with EGFR inhibitors, showing decreased phosphorylated Akt/phosphatidylinositol 3-kinase pathway activation. This action has a key role in cell survival and keratinocyte differentiation [6] in both the epidermis and the dermis, and also upregulation of p27 in the epidermis of patients treated with cetuximab, probably leading to growth inhibition of basal keratinocytes [7]. Moreover, treatment with EGFR inhibitors induces early differentiation by upregulating the expression of terminal differentiation markers (keratin 1 and keratin 10) [8]. Another mechanism described during keratinocyte differentiation is the activation of signal transducer and activator of transcription 3 in the basal layer of the epidermis [9], but there are no data regarding the association between this finding and the skin toxicity caused by EGFR inhibitors. This could result in greater alterations in both the epidermis and follicles such as atrophy, which is seen in
Fig. 1 Examples of cutaneous toxicities: in the clockwise direction, overgrowth of eyelashes, paronychia, skin lesions on the back, and facial rash

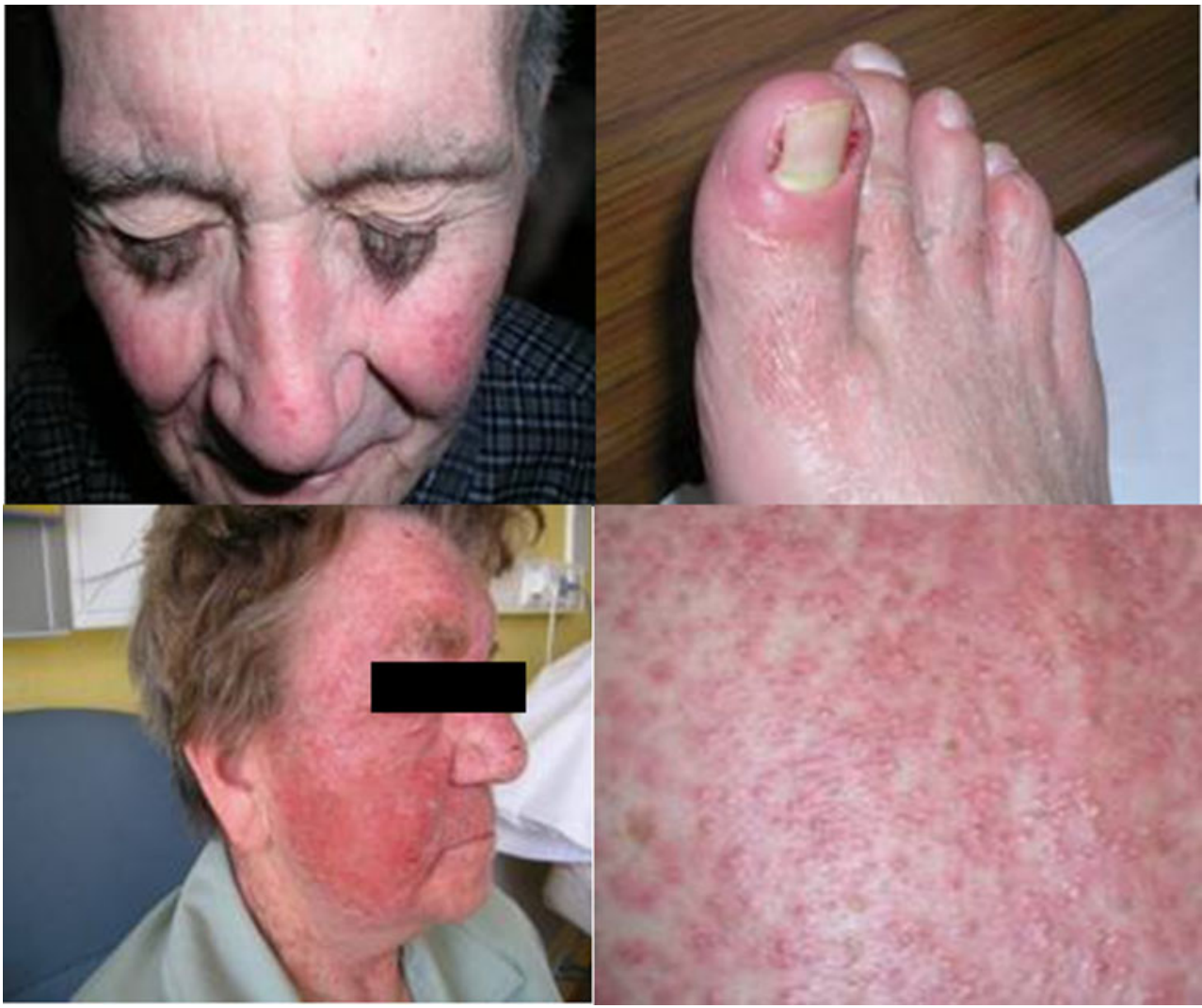


$87.5 \%$ of patients treated with cetuximab and in $50 \%$ of patients treated with panitumumab, but also dyskeratosis, dysmaturation, and suppurative folliculitis are quite frequent, whereas dermal inflammatory infiltrate is less frequent with cetuximab than with panitumumab. This is probably due to the lower levels of CD68, CD54, and CD4 seen in these patients [10 $]$. In addition, EGFR inhibitors induce lower expression of cytoskeletal proteins - vinculin and $\alpha_{1}$-actinin - reducing migration and invasion [11], whereas in keratinocytes they increase chemokine expression by upregulating extracellularsignal-regulated kinases 1 and 2. This process, resulting in enhanced skin inflammation [12], is probably not mediated by the arachidonic acid/prostaglandin pathway, with recruitment of leukocytes that release enzymes, causing apoptosis and tissue damage, which is responsible for clinical cutaneous manifestations. These clinical findings are also know as PRIDE (papulopustules and or paronychia, regulatory abnormalities of hair growth, itching, and dryness due to EGFR inhibitors) [13].

\section{Clinical Aspects}

Clinical signs and symptoms may be divided into three categories depending on their target: inflammation of the pilosebaceous follicle, represented by skin rash associated with EGFR inhibitors, which occurs at an early stage and is frequent; alteration of the skin barrier, which is primarily responsible for xerosis, fissures, and pruritus, which are frequent and delayed; and lesions of the skin appendages (paronychia, pyogenic granuloma, hair changes), which are delayed and less frequent [14•].

Skin rash is the commonest dermatological toxicity, and affects $85 \%$ of patients treated with cetuximab [15] and $90 \%$ of patients treated with panitumumab [16]. It usually develops early during the first week or first month of therapy. Thereafter, it tends to improve spontaneously despite continued treatment. It is often confined to seborrheic areas (rich in sebaceous glands): the mid-facial region, excluding the periocular region and the upper trunk (typically V-shaped), often extending to retroauricular areas, the scalp, the nape of the neck, shoulders, and even the pubis. In some patients it may involve the extremities $[17,18]$. Clinically, it is characterized by a monomorphic papulopustular eruption which consists of erythematous follicular papules that evolve into pustules, sometimes coalescent, thus forming inflammatory plaques, which may become infected, usually with Staphylococcus aureus, and form yellow crusts [7]. However, evolution from pustules to crust formation is not necessarily a sign of infection, but corresponds to the drying out of the exudates on the surface of the epidermis. Comedones and hyperseborrhea do not occur [19]. Histological analysis reveals a superficial inflammatory cell infiltrate around the follicular infundibulum. Later, an influx of neutrophils causes the rupture of the follicle and the epithelial lining [20].
This histological picture corresponds to superficial aseptic neutrophilic suppurative folliculitis associated with atrophy of the stratum corneum. The pustular appearance and the clinical and histological differences suggest that this rash is not acne. So terms such as "acneiform" and "acne-like" should be avoided.

Skin xerosis is present in up to $35 \%$ of patients receiving EGFR inhibitor therapy. Unlike skin rash, it generally has a late onset after around 30-60 days or more but persists throughout treatment with EGFR inhibitors. It manifests itself in the form of dry, squamous skin, often accompanying or following papulopustular rash. The xerosis may evolve to chronic asteatotic eczema (in one third of cases), and may be associated with painful fissures at the fingertips, palms, or knuckles and on the soles, which may become infected. Some patients experience dryness of the vagina and perineum, causing discomfort [17]. In physiopathological terms, skin xerosis seems to be linked to the abnormal differentiation of keratinocytes, which is associated with a reduction in the synthesis of loricrin - a protein located in the corneocyte envelope and which plays a key role in maintaining the integrity of the skin barrier [21].

Pruritus occurs in about half of all patients treated with EGFR inhibitors, having a relatively strong impact on their health-related quality of life. Generally, it is associated with papulopustular rash, but can also occur without rash, as a consequence of dry skin [22]. Pruritus caused by the chronicity of xerosis can also continue by an increase in the number of dermal mast cells - histamine-releasing cells-identified during EGFR inhibitor treatment [23].

Nail and periungual toxicity occur in $10-20 \%$ of patients in general after 2 months or more of EGFR inhibitor therapy and consist mainly of nail fold inflammation (paronychia) characterized by a red, warm, painful border around the nail, sometimes associated with serous discharge. Periungual pyogenic granuloma-like lesions may complicate paronychia, leading to pain and functional limitations. Also onycholysis or onychodystrophy may result as a consequence of nail matrix inflammation [22]. This clinical presentation may be aggravated by infection due to $S$. aureus, Gram-negative bacilli, or more rarely, Candida albicans. It must be suspected if the patient experiences intense, pulsatile pain and a buildup of pressure and if crusts appear [24]. The other ungual anomalies reported refer to the stoppage or slowing of nail growth, fine, fragile, or brittle nails, and onycholysis (detachment of the ungual bed of a distolateral origin) [13].

Hair changes usually occur 2-5 months after the start of treatment. They occur in approximately $50 \%$ of patients treated with EGFR inhibitors, and may include trichomegaly and hypertrichosis of eyelashes, often presenting as facial hirsutism, and also the texture of the facial and scalp hair can change and become wavy, fine, and brittle. Slower growth and alopecia are regularly observed. They generally resolve spontaneously after discontinuation of therapy [17]. 
Finally, the commonest oral complication is mucositis, presenting as broad areas of erythema or aphthous-like stomatitis [25], whereas severe mucositis and other oral side effects are infrequent.

\section{Management of Skin Toxicity}

Although skin toxicity is the most frequent side effect and impacts a very large number of patients with CRC treated with EGFR inhibitors, there are only a few controlled studies in the literature to guide best practice for management. Mostly they are based on the experience of clinicians, case reports, and nonrandomized studies with a small sample size. In the absence of definitive evidence from randomized trials, the Multinational Association of Supportive Care in Cancer (MASCC) has recently reviewed pertinent studies in the literature using established criteria in order to develop first-generation recommendations for dermatological toxicities associated with EGFR inhibitors [22]. An group of Italian experts has presented recommendations for the management of each type of skin toxicity during treatment with cetuximab, on the basis of a review of literature, to improve compliance and outcomes of patients treated with EGFR inhibitors [26].

The reactive treatment of skin rash depends on toxicity severity, evaluated by version 4.0 of the National Cancer Institute (NCI) Common Terminology Criteria for Adverse Events (CTCAE) scale, which is the reference severity scale used in clinical trials to classify cutaneous side effects related to EGFR inhibitors. For grade 1 (characterized by papulopustules or symptom-free erythema), no specific treatment is recommended, whereas grade 2 (characterized by eruption with papules or pustules covering less than $50 \%$ of the body surface, with symptoms that do not interfere with daily activities) can be treated with topical antibiotherapy (clindamycin $1 \%$ gel, erythromycin $3 \%$ gel, or metronidazole $1 \%$ gel) two times a day until it improves to grade 1, and for the pustule-prevalent type, orally administered tetracycline (minocycline $100 \mathrm{mg} /$ day, doxycycline $100 \mathrm{mg}$ /day) can be used for 4 weeks. For grade 3 toxicity (characterized by eruption with papules or pustules covering more than $50 \%$ of the body surface, with symptoms that interfere with daily activities) it is recommended to interrupt treatment for less than 21 days and if no improvement occurs to discontinue therapy. Moreover, topical treatment as for grade 2 should be used together with systemic treatment with orally administered tetracycline for 4 weeks and oral corticosteroids (prednisone $0.5 \mathrm{mg} / \mathrm{kg}$ ) for up to 10 days. For nonresponsive and grade 4 patients (generalized rash or severe symptoms that require emergency treatment), systemic treatment with an oral retinoid (isotretinoin $0.3-0.5 \mathrm{mg} / \mathrm{kg}$ ), intravenous corticosteroids, intravenous antibiotics (amoxicillin/ clavulanic acid, gentamicin), intravenous antihistamines, and hydration or hospitalization can be considered. However, a new classification system specifically for side effects related to EGFR inhibitors has been put forward by a group of experts, the MASCC, and is based on the severity of local involvement of folliculitis and not on the degree of extension [27]. A recent prospective study comparing this classification system (MESTT) with version 4.0 of CTCAE has shown that the correlation is excellent for rash, pruritus, xerosis, onycholysis, and alopecia, but there is an increase in toxicity grade with the MESTT classification for paronychia [28•]. Although the MESTT classification seems to be more precise, it is also more difficult to put into daily practice. Moreover, it has still to be validated. In contrast, even though it is not yet sufficiently appropriate for assessing the skin toxicity of EGFR inhibitors, version 4.0 of CTCAE has already been validated and is widely used in current practice.

Interesting data have been presented on the beneficial effect of a vitamin $K_{1}$ cream in prevention and treatment of skin rash induced by EGFR inhibitors. A reduction of the severity of skin rash related to EGFR inhibitors to grade 1 in 2.3 weeks [29], a lower incidence of skin rash of all grades, and the absence of severe forms when vitamin $\mathrm{K}_{1}$ cream was used for prophylaxis were reported [30]. All published reports are, however, based on studies without a control group.

In the prophylactic treatment, $1 \%$ hydrocortisone combined with moisturizer sunscreen and $100 \mathrm{mg}$ minocycline twice daily for the first 6 weeks or $100 \mathrm{mg}$ doxycycline twice daily for the first 8 weeks are recommended. This is supported by randomized phase III trials which demonstrated a significant decrease of skin rash severity [31, 32]. A recent phase II randomized trial [33•] comparing preemptive skin toxicity therapy with reactive treatment in 115 patients with CRC treated with panitumumab combined with irinotecan or irinotecan plus infusional 5-fluorouracil/leucovorin (FOLFIRI) has shown a significant reduction of approximately $50 \%$ in the incidence of toxicities greater than grade 2 in favor of the preemptive group versus the reactive group [29\% vs $62 \%$; hazard ratio (HR) 0.3]. This preemptive strategy had no impact on the efficacy of the antitumoral treatment. There were no significant differences in overall response rate, disease control rate, and median progression-free survival (PFS) (4.7 months vs 4.1 months; HR 1). On the basis of the high frequency of skin rash and the early development during treatment with EGFR inhibitors, preventive/prophylactic management can be recommended [22].

No randomized trials studying the prevention or treatment of skin xerosis are in progress. Preventive strategies are important, including the use of tepid water, bath oils, or mild moisturizing soaps without fragrances for bathing. It is also important to avoid alcohol-containing lotions and direct sun exposure [34]. Moderate xerosis may be treated using moisturizing and emollient creams which contain urea, colloid oatmeal, or ammonium lactate and lactic acid for scaly areas. For severe xerosis, topical steroid creams may be necessary 
$[35,36]$. If there are fissures, thick moisturizing or zinc oxide creams may be applied. Steroid tape and hydrocolloid dressing are recommended for painful erythematous areas [37].

Even though there are no clinical trials in the literature evaluating treatment of pruritus induced by EGFR inhibitors, current guidelines recommend nonsedating secondgeneration antihistamines as a first approach, and an antiepilectic agent such as pregabalin or gabapentin as second-line treatment. Recent evidence has shown the efficacy of aprepitant in reducing erlotinib-induced pruritus [38], but no evidence exists for other EGFR inhibitors.

Paronychia is a difficult side effect to treat and mostly occurs after several months of treatment. There are no randomized trials investigating treatment of paronychia. Recommendations are based on experience, expert opinion, and case reports. It is very important to minimize periungual trauma (wearing comfortable shoes and avoiding aggressive pedicure), decrease inflammation, and prevent superinfection (use of topical corticosteroids and an anti-inflammatory dose of tetracycline is recommended) [39-46].

In the management of hirsutism, topically administered eflornithine cream has been studied in a randomized clinical trial. It was well tolerated and resulted in a significant improvement of quality of life [47]. Minoxidil has been found to be effective in treating nonscarring alopecia. Topically administered hydrocortisone $(0.2 \%)$, steroid shampoo, and class 1 steroid lotions [19] and mild shampoo followed by antibiotic spray have recently been reported to be effective in prevention and management aimed at reducing inflammation in scarring alopecia [48] .

Finally, the management of mucositis induced by EGFR inhibitors is based on the MASCC guidelines. These recommend assessing the oral mucosa prior to and during treatment. Mucositis-associated pain is aggressively treated with topical and systemic analgesics and sometimes opioids. Moreover, minocycline and doxycycline may be indicated for management of oral aphthous lesions [49]. Specific treatment is needed if specific oral infections such as candidiasis, herpes virus reactivation, and mucositis are diagnosed.

In conclusion, skin toxicity is a common adverse effect of EGFR-targeted agents. Current data indicate that both the onset and the intensity of rash are related to drug exposure, although the cause of rash remains unclear. Skin toxicity does not affect all patients and there is a high level of interpatient variability. This could be due to the methods used to assess and categorize rash. Susceptibility to both rash and the pharmacological effects of EGFR-targeted agents could be linked to polymorphic variations in the EGFR gene [1].

\section{Relationship Between Skin Rash and Efficacy}

Several studies have investigated the relationship between rash and clinical outcome with both cetuximab and panitumumab.
Patients with metastatic CRC who developed skin rash during treatment with EGFR inhibitors had a better outcome than those who had grade 0 rash. Particularly, Saltz et al. [50] reported that $19.25 \%$ of patients treated with cetuximab in combination with irinotecan had no rash and a median survival of 4.1 months, compared with 6.2 months for those with grade 1 rash, 10.5 months for those with grade 2 rash, and 14.9 months for those with grade 3 rash (no rash vs grade 3 rash, $p<0.0001)$. Similar results were observed in patients treated with cetuximab alone [51]. In a randomized phase II trial of cetuximab plus irinotecan versus chemotherapy alone, $9.4 \%$ of patients receiving the experimental treatment developed grade 3 rash, compared with $5.2 \%$ of patients in the control arm. In addition, a higher response rate and median survival was associated with the severity of rash [52]. The EVEREST trial is based on this evidence, and showed a strong association between skin rash and clinical outcome in patients with metastatic CRC receiving cetuximab treatment (and also the reported relationship between rash severity and drug dose exposure) $[1,24,38,53]$. The EVEREST trial investigated the effect of cetuximab dose escalation in those who developed no or mild skin reaction after 21 days of treatment with irinotecan plus cetuximab at a standard dose. Dose escalation was associated with higher intensity of rash of grade higher than 2 (59\% vs $38 \%$ ), and higher disease control rate (70\% vs $58 \%$ ) for K-Ras wild-type patients compared with the standard dose. This effect suggests that cetuximab dose escalation improves the efficacy of therapy in patients with no or minimal skin rash [54]. Also for panitumumab a significant relationship between skin rash severity and clinical outcome has been reported. Skin rash was observed in about $90 \%$ of patients with metastatic CRC treated with panitumumab compared with $9 \%$ of patients in the best support group [16]. Among patients in the panitumumab arm, $86 \%$ of responders had a maximum skin rash severity of grade $2-4$, and the remaining $14 \%$ of responders had a grade 1 rash. PFS and overall survival were better for patients with grade $2-4$ rash than for patients with grade 1 rash (PFS HR 0.62; overall survival HR 0.59). A subsequent trial which considered the K-Ras mutation status of patients confirmed the association between skin rash severity as measured by both the CTCAE grading system and dermatology life quality index scores and PFS, overall survival, disease-related symptoms, and health-related quality of life only for K-Ras wild-type patients treated with panitumumab, and not for the mutant K-Ras group [55]. Although skin rash is a marker of clinical benefit in patients with metastatic CRC treated with EGFR inhibitors, its use as a predictor factor of response in clinical practice has several limitations: it was not possible to select patients who may derive greater benefit from EGFR inhibitor therapy prior to the treatment; moreover, only a subgroup of patients who developed skin rash during EGFR treatment derived a significant survival benefit from therapy, probably because the amount of drug required to cause rash is 
less than that required to induce tumor inhibition [56]. Finally, a direct relationship between EGFR blockade and skin rash development could not be the only possible explanation for this clinical association. For example, EGFR polymorphisms might increase the genetic susceptibility of some patients to develop rash and tumor response independently as described by Perea et al. [57]. Skin rash could be considered a factor correlated with a better prognosis rather than a predictor of response. It might be that the occurrence of rash reflects a patient's ability to develop an inflammatory reaction in response to damage caused by EGFR inhibitors in the skin, irrespective of the effect of EGFR inhibition in the tumor. Therefore, since our understanding of cause of rash remains limited, more studies on this are required.

\section{Hypomagnesemia}

Hypomagnesemia is a frequent adverse event (often ignored in many studies) reported during treatment with EGFR inhibitors, both with cetuximab (Erbitux ${ }^{\circledR}$; ImClone Systems, New York, NY, USA) and with panitumumab [58]. It has not been described with small molecules targeting the EGFR pathway, such as gefitinib and erlotinib. It can be considered as an antibody-specific phenomenon. Schrag et al. [59] first reported on a patient with cetuximab-induced hypomagnesemia associated with significant clinical symptoms as fatigue and paresthesias. A recent meta-analysis [60] revealed that $36.5 \%$ of patients treated with cetuximab for various cancers had hypomagnesemia of any grade, and 5.6\% had grade 3-4 hypomagnesemia. A similar result can be observed in the meta-analysis of Petrelli et al. [61], which included only prospective randomized phase III trials comparing cetuximab or panitumumab with standard chemotherapy or best supportive care. It showed a significantly higher risk of developing hypomagnesemia in the population treated with EGFR inhibitors (relative risk 5.83). Finally, the meta-analysis of Nie et al. [62] also reported a significantly higher incidence of severe hypomagnesemia (grade 3-4) in patients with metastatic CRC treated with the EGFR inhibitors cetuximab and panitumumab compared with standard chemotherapy $(27.2 \%$ vs $5.6 \%$; odds ratio 6.73 ).

\section{Pharmacological Mechanism}

The mechanism behind this toxicity has been described by Groenestege et al. [63]. EGFR inhibitors are strongly expressed in the kidney, particularly in the ascending limb of the loop of Henle, where $70 \%$ of filtered magnesium is reabsorbed. EGFR blockade may interfere with magnesium transport, provoking renal wasting. The blockade of EGFR in the nephron would reversibly impair the function of the protein TRPM6, a member of the transient receptor potential family of the cation channels localized along the apical membrane of the loop of Henle and the distal convoluted tubule (where EGFR is overexpressed). This is involved in active transport of extracellular magnesium [64, 65]. Furthermore, Tejpar at al. [66] showed using 24-h urine analysis and intravenous magnesium load test efficacy that patients treated with monoclonal antibody EGFR inhibitors had defects in renal magnesium reabsorption. However, there is no direct evidence to support this hypothesis. The effects of EGFR blockade on magnesium absorption from the gut (where EGFR and TRPM6 are expressed) cannot be excluded. The same hypothesis of "renal tubular damage" was previously used to explain the phenomenon of "hypomagnesemic hypocalcemia," which was subsequently attributed to parathyroid hormone unresponsiveness in the presence of low serum magnesium levels [67]. According to this hypothesis, hypocalcemia is a secondary effect of low serum magnesium levels via peripheral blockade of parathyroid hormone. Correction of hypomagnesemia would restore parathyroid hormone responsiveness, resulting in a restoration of the equilibrium [68]. Finally, a homolog of TRPM6 protein, TRPM7, has recently been associated not only with magnesium homeostasis, but also with CRC pathogenesis and tumor growth [69]. This might explain a possible linkage between hypomagnesemia related to EGFR inhibitors and tumor progression, which needs to be explored in further studies.

\section{Clinical Aspects}

Hypomagnesemia may be divided into different clinical severity classes according to CTCAE version 4.0. The hypomagnesemia observed during treatment with EGFR inhibitors is mostly grade $1-2$. Patients are often completely asymptomatic or have minimal symptoms such as fatigue. High-grade hypomagnesemia (grade 3, magnesium concentration between 0.7 and $0.9 \mathrm{mg} / \mathrm{dl}$; grade 4, magnesium concentration below $0.7 \mathrm{mg} / \mathrm{dl}$ ) has been reported in 10-15\% of patients treated with cetuximab, and in 3-6\% of patients treated with panitumumab [16]. The respective risk seems to be associated with the duration of treatment $(6 \%, 23 \%$, and $47 \%$ in patients receiving cetuximab treatment for less than 3 months, for 3-6 months, and for more than 6 months, respectively) [68]. Severe hypomagnesemia is generally associated with nonspecific symptoms such as irritability, paresthesias, somnolence, confusion, temporospatial disorientation, and severe fatigue. These symptoms could easily be attributed to the underlying tumor or to previous/concomitant chemotherapy [70]. Magnesium is an important component of GTPase and a cofactor for $\mathrm{Na} / \mathrm{K}$-ATPase, adenylyl cyclase, and kinases. As a result, severe hypomagnesemia can induce cardiac arhythmia, coronary artery vasospasm, and sudden death. Appropriate and aggressive replacement of magnesium is recommended [71]. 


\section{Management}

Treatment of severe hypomagnesemia consists of intravenous supplementation of magnesium at doses of 6-10 g $\mathrm{MgSO}_{4}$ daily or two to three times a week. Oral supplementation is mostly insufficient. Magnesium supplementation also results in normalization of serum calcium levels. Discontinuation of EGFR inhibitor therapy led to the resolution of hypomagnesemia in all cases [68]. However, in all patients receiving EGFR inhibitor treatment, magnesium levels should be monitored every 4-8 weeks until 48 weeks after the last therapy.

\section{Relationship Between Toxicity and Efficacy}

Some trials explored the relationship between hypomagnesemia related to EGFR inhibitors and the clinical efficacy of therapy, showing conflicting results. A recent retrospective analysis [72] of 68 patients with metastatic CRC treated with cetuximab and irinotecan showed a higher response rate, a longer time to progression, and longer overall survival in 25 patients, with more than $20 \%$ decrease in magnesium levels compared with those patients with minor decrease of serum magnesium levels during treatment (response rate $64 \%$ vs $25.6 \%, p=0.04$; time to progression 6 months vs 3.6 months, $p=0.0001$; overall survival 10.7 months vs 8.9 months, $p=0.021$ ). A similar result was observed in a subsequent trial [73••] with 143 patients with K-Ras wild-type metastatic CRC receiving the same therapy (irinotecan and cetuximab). Patients with an early decrease of magnesium levels of more than $50 \%$ compared with the basal level had a higher tumor response $(55.8 \%$ vs $16.7 \%, P<0.0001$ ), a longer time to progression (6.3 months vs 3.6 months, $P<0.0001)$, and a longer median overall survival (11.0 months vs 8.1 months, $P=0.002$ ). However, another retrospective analysis [74], exploring the association between hypomagnesemia and outcome in the NCIC Clinical Trials Group/Australasian Gastro-Intestinal Trials Group CO.17 clinical trial, showed that higher grade of hypomagnesemia and greater percent reduction in magnesium concentration at day 28 predict worse survival in patients treated with cetuximab in both the K-Ras wildtype population and the mutant population. We have to conclude that the results are conflicting.

\section{Gastrointestinal Toxicity}

Gastrointestinal toxicity is frequent during EGFR inhibitor therapy, including nausea and vomiting, abdominal pain, constipation, and diarrhea. Mostly, mild to moderate grades are reported; in $2-3 \%$ of patients it can be severe. A significantly higher incidence of severe diarrhea (about 15-17 \% of patients) has been reported when cetuximab or panitumumab is given in combination with chemotherapy, particularly the irinotecan regimen (Crystal trial [75], $15 \%$ vs $10.5 \%$; and Peeters trial [76], $14 \%$ vs $10 \%$ ), even though the same incidence of grade 3-4 diarrhea was observed with the addition of panitumumab to the combination of leucovorin, 5fluorouracil, and oxaliplatin [77••]. Finally, a recent metaanalysis [62] showed a significant increase of grade 3-4 adverse events with EGFR inhibitors plus chemotherapy compared with chemotherapy alone in patients with metastatic CRC, including severe diarrhea $(62.3 \%$ vs $55.7 \%$; odds ratio 1.36). At present, the exact mechanism behind this toxicity is not clearly understood. It seems to be associated with blockade of EGFR activity in the gastrointestinal tract. EGFR is overexpressed and plays an important role in cell proliferation and differentiation. It also regulates cation channels involved in active transport of extracellular ions and intestinal secretions. Furthermore, the STEPP trial [33•], which explored the impact of preemptive treatment with doxycycline in patients with metastatic CRC, showed a significant reduction of both skin and nonskin toxicity (such as diarrhea and dehydration) in the preemptive group compared with the reactive control group, suggesting that diarrhea induced by the combination of EGFR inhibitors and chemotherapy has an inflammatory or infectious component that is potentially improved with the use of doxycycline, the only systemic agent administered. However, further studies are needed to evaluate the use of doxycycline for treatment of diarrhea induced by the combination of EGFR inhibitors and chemotherapy. Management of severe diarrhea should be aggressive, including use of loperamide or diphenoxylate, rehydration, and electrolyte replacement. Sometimes hospitalization is required.

\section{Infusion Reaction}

Even though infusion reaction is not a frequent side effect observed during EGFR inhibitor therapy, it is considered the most life-threatening side effect. Infusion reactions of any grade have been reported in about $10 \%$ of patients treated with cetuximab and in $4 \%$ of patients treated with panitumumab. Severe reactions (grade 3-4, NCI Common Toxicity Criteria, version 2) occurred in $4 \%$ of patients treated with cetuximab and in less than $1 \%$ of patients treated with panitumumab. In contrast to panitumumab therapy, cetuximab therapy requires premedication prior to infusion. Most severe reactions with cetuximab usually occur a few minutes after the first infusion, but $33 \%$ may be observed after the second dose, and rarely with subsequent therapies. The physiopathological mechanism underlying a severe infusion reaction seems to be associated with IgE- 
mediated anaphylaxis, which would affect patients who had $\operatorname{IgE}$ antibodies prior to treatment, as shown by Chung et al. [78]. In this trial, IgE antibodies against cetuximab were detected in blood samples of $68 \%$ of patients who had infusion reaction, compared with $2 \%$ of those without reaction $(p<0.001)$. Moreover, $\operatorname{IgE}$ antibodies were discovered to be specific for $\alpha-1,3$-galactose, expressed on the Fab portion of cetuximab heavy chain. So subsequent reexposure to the antigen would cross-link the Fab portion of IgE molecules, activating the mast cells or basophils and triggering the release of clinical mediators. A phase III trial of panitumumab showed that neutralizing antibodies were detected in only $1.4 \%$ of patients who had an infusion reaction. The role of these in the pathogenesis of reactions and the effective mechanism behind this have yet to be understood. The management of mild-to-moderate infusion reaction consists of prompt interruption of infusion and antihistamine administration. After remission of clinical symptoms, the treatment can be continued at $50 \%$ of the standard rate. Premedication with antihistamines and steroids is mandatory prior to the first cetuximab therapy, and is strongly recommended prior to subsequent therapies. It seems to reduce the cetuximab-related infusion reactions as shown by Siena et al. [79•]. No premedication is recommended prior to panitumumab therapy. Finally, Nielsen et al. [80] described two patients with grade 2 reaction who were rechallenged with cetuximab. Antihistamines and steroids prior to the next therapy were given without reaction. There are also limited data on rechallenge with an alternative EGFR inhibitor. Rechallenge with panitumumab without any premedication in patients who during infusion developed hypersensitivity reactions to cetuximab (11 cases) and vice versa (two cases) [81] showed no evidence of acute reaction $[82,83]$.

\section{Pulmonary Toxicity}

Adverse pulmonary reactions related to EGFR inhibitors are rare. As discussed already, these drugs can trigger a hypersensitivity reaction during drug infusion. In the case of respiratory symptoms, anaphylactic reactions have been observed with rapid onset of airway obstruction (bronchospasms, stridor, hoarseness). The use of premedication is useful in preventing these episodes. Severe reactions require immediate interruption of infusion and use of bronchodilators, antihistamines, and corticosteroids. The incidence of severe infusion reactions is approximately $3 \%$ with cetuximab and $0.1 \%$ with panitumumab [84].

Another clinical entity described with the use of cetuximab is bronchiolitis [85]. Pulmonary fibrosis has been rarely associated with cetuximab and panitumumab [86].

\section{Conclusions}

EGFR inhibition is one of the most important strategies for the treatment of patients with metastatic CRC. The introduction of the monoclonal antibodies cetuximab and panitumumab both as a single agent and in combination with chemotherapy has resulted in improvement of clinical outcomes in this setting. These advantages often include improvement in quality of life, but this effect could be compromised by toxicities related to EGFR inhibitors.

Skin toxicity is the most frequent side effect observed. It seems to be related to the expression of EGFR in the skin tissue. Some strategies are known to prevent and manage its onset. It does not usually require interruption of treatment except for severe manifestations with complications. However, proper management is essential to avoid delay or suspension of treatment and impairment of quality of life. Some studies have highlighted a positive relationship between skin rash and clinical outcomes.

Hypomagnesemia is related to the effect of EGFR function on magnesium reabsorption in the kidney. Its management consists of magnesium supplementation only in severe cases.

Gastrointestinal toxicity, such as diarrhea, and thromboembolic events can be a consequence of chemotherapy alone. In the treatment regimens including the combination of chemotherapy and the anti-EGFR monoclonal antibodies, a synergism has been recognized for this toxicity.

Finally, infusion reactions are rare events, but can be lethal. Cetuximab seems to be more associated with these events than panitumumab. An IgE-mediated anaphylaxis could explain this phenomenon. Management needs to be immediate since the onset is usually sudden. The opportunity of rechallenge after these events is under discussion.

Only in a few cases does the anti-EGFR-drug-related toxicity lead to withholding of treatment. Rarely, it leads to other severe complications. For this reason, proper management of these toxicities is mandatory to achieve full delivery of planned treatment and to not compromise quality of life.

\section{Compliance with Ethics Guidelines}

Conflict of Interest Christian Rolfo declares he has no conflict of interest.

Giuseppe Bronte declares he has no conflict of interest.

Francesco Passiglia declares he has no conflict of interest.

Konstantinos Papadimitriou declares he has no conflict of interest. Antonio Russo declares he has no conflict of interest.

Marc Peeters has received a consulting fee/honorarium from Sanofi, Roche, and Amgen, has received compensation for a board membership from Amgen and Sanofi, is supported by a grant from Amgen, and has received payment for lectures, including service on speakers bureaus, from Amgen and Sanofi. 
Human and Animal Rights and Informed Consent This article does not contain any studies with human or animal subjects performed by any of the authors.

\section{References}

Papers of particular interest, published recently, have been highlighted as:

- Of importance

- Of major importance

1. Perez-Soler R, Saltz L. Cutaneous adverse effects with HER1/EGFRtargeted agents: is there a silver lining? J Clin Oncol. 2005;23:523546.

2. • Joshi SS, Ortiz S, Witherspoon JN, et al. Effects of epidermal growth factor receptor inhibitor-induced dermatologic toxicities on quality of life. Cancer. 2010;116:3916-23. This is one of few studies evaluating the impact of skin toxicity on quality of life by specific questionnaires.

3. Boone SL, Rademaker A, Liu D, et al. Impact and management of skin toxicity associated with anti-epidermal growth factor receptor therapy: survey results. Oncology. 2007;72:152-9.

4. Giovannini M, Gregorc V, Belli C, et al. Clinical significance of skin toxicity due to EGFR-targeted therapies. J Oncol. 2009;2009:849051.

5. Surguladze D, Deevi D, Claros N, et al. Tumor necrosis factoralpha and interleukin-1 antagonists alleviate inflammatory skin changes associated with epidermal growth factor receptor antibody therapy in mice. Cancer Res. 2009;69:5643-7.

6. Thrash BR, Menges CW, Pierce RH, McCance DJ. AKT1 provides an essential survival signal required for differentiation and stratification of primary human keratinocytes. J Biol Chem. 2006;281:12155-62.

7. Busam KJ, Capodieci P, Motzer R, et al. Cutaneous side-effects in cancer patients treated with the antiepidermal growth factor receptor antibody C225. Br J Dermatol. 2001;144:1169-76.

8. Peus D, Hamacher L, Pittelkow MR. EGF-receptor tyrosine kinase inhibition induces keratinocyte growth arrest and terminal differentiation. J Invest Dermatol. 1997;109:751-6.

9. Hauser PJ, Agrawal D, Hackney J, Pledger WJ. STAT3 activation accompanies keratinocyte differentiation. Cell Growth Differ. 1998;9:847-55

10. • Nardone B, Nicholson K, Newman M, et al. Histopathologic and immunohistochemical characterization of rash to human epidermal growth factor receptor 1 (HER1) and HER1/2 inhibitors in cancer patients. Clin Cancer Res. 2010;16:4452-60. This is a study which tries to find a relationship between histopathologic features and biological mechanisms of skin reactions.

11. Woodworth CD, Michael E, Marker D, et al. Inhibition of the epidermal growth factor receptor increases expression of genes that stimulate inflammation, apoptosis, and cell attachment. Mol Cancer Ther. 2005;4:650-8.

12. Pastore S, Mascia F, Mariotti F, et al. ERK1/2 regulates epidermal chemokine expression and skin inflammation. J Immunol. 2005; 174:5047-56.

13. Lacouture ME, Lai SE. The PRIDE (papulopustules and/or paronychia, regulatory abnormalities of hair growth, itching, and dryness due to epidermal growth factor receptor inhibitors) syndrome. Br J Dermatol. 2006;155:852-4.

14. - Peuvrel L, Bachmeyer C, Reguiai Z, et al. Semiology of skin toxicity associated with epidermal growth factor receptor (EGFR) inhibitors. Support Care Cancer. 2012;20:909-21. This is a useful guide for clinicians to describe and manage anti-EGFR-related skin toxicity.
15. Rosell R, Robinet G, Szczesna A, et al. Randomized phase II study of cetuximab plus cisplatin/vinorelbine compared with cisplatin/ vinorelbine alone as first-line therapy in EGFR-expressing advanced non-small-cell lung cancer. Ann Oncol. 2008;19:362-9.

16. Van Cutsem E, Peeters M, Siena S, et al. Open-label phase III trial of panitumumab plus best supportive care compared with best supportive care alone in patients with chemotherapy-refractory metastatic colorectal cancer. J Clin Oncol. 2007;25:1658-64.

17. Segaert S, Van Cutsem E. Clinical signs, pathophysiology and management of skin toxicity during therapy with epidermal growth factor receptor inhibitors. Ann Oncol. 2005;16:1425-33.

18. Galimont-Collen AF, Vos LE, Lavrijsen AP, et al. Classification and management of skin, hair, nail and mucosal side-effects of epidermal growth factor receptor (EGFR) inhibitors. Eur J Cancer. 2007;43:845-51.

19. Burtness B, Anadkat M, Basti S, et al. NCCN Task Force report: management of dermatologic and other toxicities associated with EGFR inhibition in patients with cancer. J Natl Compr Cancer Netw. 2009;7 Suppl 1:S5-S21. quiz S22-4.

20. Lacouture ME. Mechanisms of cutaneous toxicities to EGFR inhibitors. Nat Rev Cancer. 2006;6:803-12.

21. Miettinen PJ, Berger JE, Meneses J, et al. Epithelial immaturity and multiorgan failure in mice lacking epidermal growth factor receptor. Nature. 1995;376:337-41.

22. Lacouture ME, Anadkat MJ, Bensadoun RJ, et al. Clinical practice guidelines for the prevention and treatment of EGFR inhibitorassociated dermatologic toxicities. Support Care Cancer. 2011;19: 1079-95.

23. Gerber PA, Buhren BA, Cevikbas F, et al. Preliminary evidence for a role of mast cells in epidermal growth factor receptor inhibitorinduced pruritus. J Am Acad Dermatol. 2010;63:163-5.

24. Robert C, Soria JC, Spatz A, et al. Cutaneous side-effects of kinase inhibitors and blocking antibodies. Lancet Oncol. 2005;6:491-500.

25. Khuntia D, Harris J, Bentzen SM. Increased oral mucositis after IMRT versus non-IMRT when combined with cetuximab and cisplatin or docetaxel for head and neck cancer: preliminary results of RTOG 0234. Int J Radiat Oncol Biol Phys. 2008;72:S33.

26. Pinto C, Barone CA, Girolomoni G, et al. Management of skin toxicity associated with cetuximab treat. in combination with chemotherapy or radiotherapy. Oncologist. 2011;16:228-38.

27. Lacouture ME, Maitland ML, Segaert S, et al. A proposed EGFR inhibitor dermatologic adverse event-specific grading scale from the MASCC skin toxicity study group. Support Care Cancer. 2010; 18:509-22.

28. - Chan A, Tan EH. How well does the MESTT correlate with CTCAE scale for the grading of dermatological toxicities associated with oral tyrosine kinase inhibitors? Support Care Cancer. 2010;19:1667-74. This analysis attempts to find a correlation between two different ways of grading skin toxicity.

29. Ocvirk J, Rebersek M. Treatment of cetuximab-associated cutaneous side effects using topical application of vitamin $\mathrm{K} 1 \mathrm{cream} . \mathrm{J}$ Clin Oncol. 2009;27(15S):e15087.

30. Ocvirk J, Rebersek M, Boc M, et al. Prophylactic use of K1 cream for reducing skin toxicity during cetuximab treatment in patients with metastatic colorectal cancer (mCRC). J Clin Oncol. 2010;28 (15 Suppl):e14011.

31. Jatoi A, Rowland K, Sloan J. Does tetracycline prevent or palliate epidermal growth factor receptor (EGFR) inhibitor-induced skinrash? Results of a phase III trial from North Central Cancer Treatment Group (N03CB). J Clin Oncol. 2007;25:494s.

32. Scope A, Agero AL, Dusza SW, et al. Randomized double-blind trial of prophylactic oral minocycline and topical tazarotene for cetuximab-associated acne-like eruption. J Clin Oncol. 2007;25:5390-6.

33. - Lacouture ME, Mitchell EP, Piperdi B, et al. Skin toxicity evaluation protocol with panitumumab (STEPP), a phase II, 
open-label, randomized trial evaluating the impact of a preemptive skin treatment regimen on skin toxicities and quality of life in patients with metastatic colorectal cancer. J Clin Oncol. 2010;28:1351-7. This study evaluates for panitumumab the preemptive and reactive treatment of skin toxicity. The preemptive treatment showed a significant reduction of incidence.

34. Saif MW, Kaley K, Lamb L, et al. Management of skin toxicities of anti-EGFR agents in patients with pancreatic cancer and other GI tumors by using electronic communication: effective and convenient. JOP. 2010;11:176-82.

35. Perez-Soler R, Delord JP, Halpern A, et al. HER1/EGFR inhibitorassociated rash: future directions for management and investigation outcomes from the HER1/EGFR inhibitor rash management forum. Oncologist. 2005;10:345-56.

36. Wollenberg A, Kroth J, Hauschild A, Dirschka T. Hautreaktionen unter EGFR-Inhibitoren - Klinik und Management. Dtsch Med Wochenschr. 2010;135:149-54.

37. Hu JC, Sadeghi P, Pinter-Brown LC, et al. Cutaneous side effects of epidermal growth factor receptor inhibitors: clinical presentation, pathogenesis, and management. J Am Acad Dermatol. 2007;56:317-26.

38. Roe E, Garcia Muret MP, Marcuello E, et al. Description and management of cutaneous side effects during cetuximab or erlotinib treatments: a prospective study of 30 patients. J Am Acad Dermatol. 2006;55:429-37.

39. Wollina U. Acute paronychia: comparative treatment with topical antibiotic alone or in combination with corticosteroid. J Eur Acad Dermatol Venereol. 2001;15:82-4.

40. Rao A, Bunker C. Efficacy and safety of tacrolimus ointment $0.1 \%$ vs. betamethasone 17 -valerate $0.1 \%$ in the treatment of chronic paronychia: an unblinded randomized study. $\mathrm{Br} \mathrm{J}$ Dermatol. 2010;163:208. author reply 208-9.

41. Rigopoulos D, Gregoriou S, Belyayeva E, et al. Efficacy and safety of tacrolimus ointment $0.1 \%$ vs. betamethasone 17 -valerate $0.1 \%$ in the treatment of chronic paronychia: an unblinded randomized study. Br J Dermatol. 2009;160:858-60.

42. Daniel III CR, Daniel MP, Daniel CM, et al. Chronic paronychia and onycholysis: a thirteen-year experience. Cutis. 1996;58:397401 .

43. Rosenbaum D, Merenstein D, Meyer F. Topical steroids more effective than antifungals for chronic paronychia. J Fam Pract. 2002;51:824.

44. Roberts DT, Richardson MD, Dwyer PK, Donegan R. Terbinafine in chronic paronychia and candida onychomycosis. J Dermatol Treat. 1992;S1:39-42.

45. Rutala WA, Weber DJ. Uses of inorganic hypochlorite (bleach) in health-care facilities. Clin Microbiol Rev. 1997;10:597-610.

46. Shu KY, Kindler HL, Medenica M, Lacouture M. Doxycycline for the treatment of paronychia induced by the EFGR inhibitor cetuximab. Br J Dermatol. 2006;154:191-2.

47. Hoffmann R. A 4-month, open-label study evaluating the efficacy of eflornithine $11.5 \%$ cream in the treatment of unwanted facial hair in women using TrichoScan. Eur J Dermatol. 2008;18:65-70.

48. Ocvirk J, Cencelj S. Management of cutaneous side-effects of cetuximab therapy in patients with metastatic colorectal cancer. J Eur Acad Dermatol Venereol. 2010;24:453-9.

49. Gorsky M, Epstein J, Raviv A, et al. Topical minocycline for managing symptoms of recurrent aphthous stomatitis. Spec Care Dentist. 2008;28:27-31.

50. Saltz L, Rubin MS, Hochster H, et al. Acne-like rash predicts response in patients treated with Cetuximab (IMC-C225) plus Irinotecan (CPT-11) in CPT-11-refractory colorectal cancer (CRC) that expresses epidermal growth factor receptor (EGFR). Clin Cancer Res. 2001;7:3766s.

51. Saltz LB, Meropol NJ, Loehrer Sr PJ, et al. Phase II trial of cetuximab in patients with refractory colorectal cancer that expresses the epidermal growth factor receptor. J Clin Oncol. 2004;22:1201-8.

52. Cunningham D, Humblet Y, Siena S, et al. Cetuximab (C225) alone or in combination with irinotecan (CPT-11) in patients with epidermal growth factor receptor (EGFR)-positive, irinotecan refractory metastatic colorectal cancer (MCRC). Proc Am Soc Clin Oncol. 2003;22:252.

53. Segaert S, Chiritescu G, Lemmens L, et al. Skin toxicities of targeted therapies. Eur J Cancer. 2009;45 Suppl 1:295-308.

54. Van Cutsem E, Peeters M, Gelderblom H, et al. Cetuximab dose escalation in MCRC patients with no or slight rash skin reactions on standard treatment (Everest). Ann Oncol. 2007;18 Suppl 7:O-0034.

55. Peeters M, Siena S, Van Cutsem E, et al. Association of progression-free survival, overall survival, and patient-reported outcomes by skin toxicity and KRAS status in patients receiving panitumumab monotherapy. Cancer. 2009;115:1544-54.

56. Walon L, Gilbeau C, Lachapelle JM. Éruptions acnéiformes induites par le cétuximab. Ann Dermatol Venereol. 2003;130:443-6.

57. Perea S, Oppenheimer D, Amador M. Genotypic bases of EGFR inhibitors pharmacological actions. Proc Am Soc Clin Oncol. 2004;196. Abstr 3005.

58. Berlin J, Neubauer M, Swanson P, et al. Panitumumab antitumor activity in patients (pts) with metastatic colorectal cancer (mCRC) expressing $<10 \%$ epidermal growth factor receptor (EGFr). J Clin Oncol. 2006;24(18S):3548.

59. Schrag D, Chung KY, Flombaum C, Saltz L. Cetuximab therapy and symptomatic hypomagnesemia. $\mathrm{J}$ Natl Cancer Inst. 2005;97:1221-4.

60. Cao Y, Liao C, Tan A, et al. Meta-analysis of incidence and risk of hypomagnesemia with cetuximab for advanced cancer. Chemotherapy. 2010;56:459-65.

61. Petrelli F, Borgonovo K, Cabiddu M, et al. Risk of anti-EGFR monoclonal antibody-related hypomagnesemia: systematic review and pooled analysis of randomized studies. Expert Opin Drug Saf. 2012;11 Suppl 1:S9-S19.

62. Nie F, Shen J, Tong JL, et al. Meta-analysis: the efficacy and safety of monoclonal antibody targeted to epidermal growth factor receptor in the treatment of patients with metastatic colorectal cancer. J Dig Dis. 2009;10:247-57.

63. Groenestege WM, Thebault S, van der Wijst J, et al. Impaired basolateral sorting of pro-EGF causes isolated recessive renal hypomagnesemia. J Clin Invest. 2007;117:2260-7.

64. Schlingmann KP, Weber S, Peters M, et al. Hypomagnesemia with secondary hypocalcemia is caused by mutations in TRPM6, a new member of the TRPM gene family. Nat Genet. 2002;31:166-70.

65. Chubanov V, Waldegger S, Mederos y Schnitzler M, et al. Disruption of TRPM6/TRPM7 complex formation by a mutation in the TRPM6 gene causes hypomagnesemia with secondary hypocalcemia. Proc Natl Acad Sci USA. 2004;101:2894-9.

66. Tejpar S, Piessevaux H, Claes K, et al. Magnesium wasting associated with epidermal-growth-factor receptor-targeting antibodies in colorectal cancer: a prospective study. Lancet Oncol. 2007;8:387-94.

67. Torralbo A, Portoles J, Perez Perez AJ, Barrientos A. Hypomagnesemic hypocalcemia in chronic renal failure. Am J Kidney Dis. 1993;21:167-71.

68. Fakih MG, Wilding G, Lombardo J. Cetuximab-induced hypomagnesemia in patients with colorectal cancer. Clin Colorectal Cancer. 2006;6:152-6.

69. Dai Q, Shrubsole MJ, Ness RM, et al. The relation of magnesium and calcium intakes and a genetic polymorphism in the magnesium transporter to colorectal neoplasia risk. Am J Clin Nutr. 2007:86:743-51.

70. Perrin C, Fabre C, Raoul JL, Boucher E. Behavioral disorders secondary to profound hypomagnesemia in a patient given cetuximab for metastatic colorectal cancer hypomagnesemia due to cetuximab treatment. Acta Oncol. 2006;45:1135-6. 
71. Fakih M. Management of anti-EGFR-targeting monoclonal antibody-induced hypomagnesemia. Oncology (Williston Park). 2008;22:74-6.

72. Vincenzi B, Santini D, Galluzzo S, et al. Early magnesium reduction in advanced colorectal cancer patients treated with cetuximab plus irinotecan as predictive factor of efficacy and outcome. Clin Cancer Res. 2008;14:4219-24.

73. • Vincenzi B, Galluzzo S, Santini D, et al. Early magnesium modifications as a surrogate marker of efficacy of cetuximab-based anticancer treatment in KRAS wild-type advanced colorectal cancer patients. Ann Oncol. 2011;22:1141-6. This is one of few studies evaluating the predictive role of early decrease in magnesium concentration for anti-EGFR efficacy. As shown in this study, hypomagnesemia is related to response rates, time to progression, and overall survival.

74. Vickers MM, Karapetis CS, Tu D, et al. Association of hypomagnesemia with inferior survival in a phase III, randomized study of cetuximab plus best supportive care versus best supportive care alone: NCIC CTG/AGITG CO.17. Ann Oncol. 2013;24:953-60.

75. Van Cutsem E, Nowacki M, Lang I, et al. Randomized phase III study of irinotecan and 5-FU/FA with or without cetuximab in the first-line treatment of patients with metastatic colorectal cancer (mCRC): The CRYSTAL trial. J Clin Oncol. 2007;25(18 Suppl):4000.

76. Peeters M, Price TJ, Cervantes A, et al. Randomized phase III study of panitumumab with fluorouracil, leucovorin, and irinotecan (FOLFIRI) compared with FOLFIRI alone as secondline treatment in patients with metastatic colorectal cancer. J Clin Oncol. 2010;28:4706-13.

77. •• Douillard JY, Siena S, Cassidy J, et al. Randomized, phase III trial of panitumumab with infusional fluorouracil, leucovorin, and oxaliplatin (FOLFOX4) versus FOLFOX4 alone as first-line treatment in patients with previously untreated metastatic colorectal cancer: the PRIME study. J Clin Oncol. 2010;28:4697-705. This is the most important study evaluating efficacy and toxicity deriving from the combination of panitumumab and chemotherapy.
78. Chung $\mathrm{CH}$, Mirakhur B, Chan E, et al. Cetuximab-induced anaphylaxis and IgE specific for galactose-alpha-1,3-galactose. $\mathrm{N}$ Engl J Med. 2008;358:1109-17.

79. - Siena S, Glynne-Jones R, Adenis A, et al. Reduced incidence of infusion-related reactions in metastatic colorectal cancer during treatment with cetuximab plus irinotecan with combined corticosteroid and antihistamine premedication. Cancer. 2010;116:182737. This study identified the proper premedication to prevent cetuximab-related infusion reactions by premedication.

80. Nielsen DL, Pfeiffer P, Jensen BV. Re-treatment with cetuximab in patients with severe hypersensitivity reactions to cetuximab. Two case reports. Acta Oncol. 2006;45(8):1137-38.

81. Saif MW, Kim R. Incidence and management of cutaneous toxicities associated with cetuximab. Expert Opin Drug Saf. 2007;6:175-82.

82. Saif MW, Peccerillo J, Potter V. Successful re-challenge with panitumumab in patients who developed hypersensitivity reactions to cetuximab: report of three cases and review of literature. Cancer Chemother Pharmacol. 2009;63:1017-22.

83. Power DG, Shah MA, Asmis TR, et al. Safety and efficacy of panitumumab following cetuximab: retrospective review of the Memorial Sloan-Kettering experience. Invest New Drugs. 2010;28:353-60.

84. Barber NA, Ganti AK. Pulmonary toxicities from targeted therapies: a review. Targ Oncol. 2011;6:235-43.

85. Chua W, Peeters M, Loneragan R, Clarke S. Cetuximabassociated pulmonary toxicity. Clin Colorectal Cancer. 2009;8(2): $118-20$.

86. Giusti RM, Shastri K, Pilaro AM, Fuchs C, Cordoba-Rodriguez R, Koti K, et al. U.S. Food and Drug Administration approval: panitumumab for epidermal growth factor receptor-expressing metastatic colorectal carcinoma with progression following fluoropyrimidine-, oxaliplatin-, and irinotecan-containing chemotherapy regimens. Clin Cancer Res. 2008;14(5):1296-302. 